\title{
Low-temperature Mössbauer study of heterosite, (Fe, $\mathrm{Mn}) \mathrm{PO}_{4}$
}

\author{
Eddy De Grave $^{\mathrm{a}, *}$, Geraldo Magela da Costa ${ }^{\mathrm{b}}$, Antoine Van Alboom ${ }^{\mathrm{a}, \mathrm{c}}$, Robert Emile Vandenberghe ${ }^{\mathrm{a}}$ \\ ${ }^{a}$ Department of Physics and Astronomy, University of Ghent, B-9000 Gent, Belgium \\ ${ }^{\mathrm{b}}$ Departamento de Química, Universidade Federal de Ouro Preto, 35400-000, Ouro Preto (MG), Brazil \\ ${ }^{\mathrm{c}}$ Faculty of Applied Engineering Sciences, University College Gent, B-9000 Gent, Belgium
}

\section{A R T I C L E I N F O}

\section{Article history:}

Received 29 October 2011

Received in revised form 18 April 2012

Accepted 3 May 2012

\section{Keywords:}

Mössbauer spectroscopy

Heterosite

Magnetic hyperfine field

Isomer shift

\begin{abstract}
A B S T R A C T
The heterosite phase occurring in a pegmatitic rock sample was characterized by X-ray diffraction, by energy-dispersive X-ray spectroscopy and by Mössbauer spectroscopy. The orthorhombic unit-cell parameters, expressed in $\AA$, were found as $a=9.733$ (1), $b=5.837$ (1) and $c=4.776$ (1). The composition was determined to be $\left(\mathrm{Fe}_{0.54} \mathrm{Mn}_{0.43} \mathrm{Mg}_{0.04}\right) \mathrm{PO}_{4}$. Mössbauer spectra recorded at temperatures $T$ of $65 \mathrm{~K}$ and higher consist of two broadened quadrupole doublets. Their isomer shifts $\delta$ are both diagnostic for the ferric state. The dominant doublet ( $\sim 60 \%$ of total area) exhibits an average quadrupole splitting $\Delta E_{\mathrm{Q} a v}$ of $1.62 \mathrm{~mm} / \mathrm{s}$ at room temperature, while the weaker broader doublet has $\Delta E_{\mathrm{Q}, \mathrm{av}}=0.68 \mathrm{~mm} / \mathrm{s}$. For temperatures $T \leq 60 \mathrm{~K}$ the spectra are composed of a broad sextet and a central quadrupole doublet. The doublet persists down to the lowest applied temperature of $17 \mathrm{~K}$. It is concluded that this doublet is due to an Fe-bearing phase other than heterosite and which gives rise to the inner doublet appearing in the spectra recorded at $T \geq 65 \mathrm{~K}$. The broad sextets, attributable to the heterosite phase, were fitted with model-independent hyperfine-field distributions. However, it was consistently experienced that using the common Lorentzian-shaped elementary sextets composing the distribution, could not adequately reproduce the observed line shapes. Instead, the calculations had to be based on the diagonalization of the complete hyperfine-interaction Hamiltonian. This is due to the unusually strong quadrupole interaction. The as-such calculated hyperfine parameters of the heterosite phase at $17 \mathrm{~K}$ may be summarized as follows: maximum-probability hyperfine field $B_{\mathrm{hf}, \mathrm{m}}=473 \mathrm{kOe}$, isomer shift $\delta_{\mathrm{Fe}}=0.54 \mathrm{~mm} / \mathrm{s}$, average quadrupole coupling constant $1 / 2 e^{2} q Q=1.50 \mathrm{~mm} / \mathrm{s}$, asymmetry parameter of the EFG $\eta=0.80$, and polar angles of the hyperfine field with respect to the EFGs principal axes frame $\Omega=40^{\circ}$ and $\Psi=90^{\circ}$. The temperature variation of the hyperfine field was interpreted in terms of the Bean-Rodbell (BR) model. The BR parameter, $\eta_{\mathrm{BR}}$, was found to be 0.90 , indicating a first-order magnetic transition at $T_{\mathrm{N}}=59.7 \mathrm{~K}$. The temperature variation of the isomer shift is explained by the second-order Doppler shift $\delta_{\text {SOD }}$. Using the Debye model for the lattice vibrational spectrum for calculating $\delta_{S O D}$, the characteristic Mössbauer temperature $\Theta_{\mathrm{M}}$ was found to be $400 \mathrm{~K}$, which is unusually low for a ferric compound.
\end{abstract}

(c) 2012 Elsevier B.V. All rights reserved.

\section{Introduction}

The mineral heterosite, $\left(\mathrm{Fe}^{3+}, \mathrm{Mn}^{3+}\right) \mathrm{PO}_{4}$, is an iron-rich member of the solid solution series heterosite - purpurite, $\mathrm{FePO}_{4}-\mathrm{MnPO}_{4}$. It is usually found as a constituent in pegmatitic rocks. The structure of a heterosite species with composition $\mathrm{Fe}_{0.65} \mathrm{Mn}_{0.35} \mathrm{PO}_{4}$ has been refined by Eventoff et al. [1]. It is of the orthorhombic olivine-type with $\mathrm{Fe}^{3+}$ and $\mathrm{Mn}^{3+}$ occupying strongly distorted corner-sharing octahedral M2 sites, which form zigzag chains running parallel to the $c$-axis. A second type of edge-sharing octahedral sites, M1, forms linear chains that are also directed along the $c$-axis. In

\footnotetext{
* Corresponding author at: Department of Physics and Astronomy, University of Ghent, Proeftuinstraat 86, B-9000 Gent, Belgium. Tel.: +32 09264 6566; fax: +3209264 6697 .

E-mail address: eddy.degrave@Ugent.be (E. De Grave).
}

isostructural triphylite, $\mathrm{Li}^{+} \mathrm{Fe}^{2+} \mathrm{PO}_{4}$, these $\mathrm{M} 1$ sites are occupied by $\mathrm{Li}^{+}$cations, while in heterosite-purpurite solid solutions they

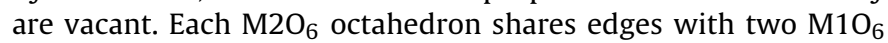
octahedra and one $\mathrm{PO}_{4}$ tetrahedron, and each $\mathrm{M}_{1} \mathrm{O}_{6}$ has common edges with two other $\mathrm{M}_{10}$ octahedra, with two $\mathrm{M}_{6} \mathrm{O}_{6}$ octahedra and with two $\mathrm{PO}_{4}$ tetrahedra. For an illustrative polyhedral view of the triphylite structure, the reader is referred to a recent report by Van Alboom et al. [2].

It is well known that ${ }^{57} \mathrm{Fe}$ Mössbauer spectroscopy is a powerful experimental technique, allowing to determine a variety of structural, electronic and magnetic properties of Fe atoms/cations present in solid materials. In that respect, the most complete information is obtained by studying the temperature dependence of the hyperfine parameters that are extracted from the Mössbauer spectra.

Mössbauer spectra recorded at room temperature (RT) for heterosites, both synthetic and natural species, have been reported 
by a number of authors [3-6]. Recently, Aldon et al. [7] presented the results of a Mössbauer study of a synthetic $\mathrm{FePO}_{4}$ sample at seven different temperatures in the range between $141 \mathrm{~K}$ and $301 \mathrm{~K}$ with the aim to evaluate the recoilless fraction of the material, the knowledge of which is useful for the estimation of the relative amount of $\mathrm{FePO}_{4}$ formed during $\mathrm{Li}$ extraction or insertion processes involving $\mathrm{Li}_{y} \mathrm{FePO}_{4}(0 \leq y \leq 1)$ systems. In the present contribution, the authors describe the results of their Mössbauer measurements carried out at many different temperatures between $15 \mathrm{~K}$ and $295 \mathrm{~K}$ for a natural heterosite species. The emphasis of this work was put towards the magnetic spectra $(T<60 \mathrm{~K})$. As far as could be traced such spectra for heterosite have so far not been reported in the literature.

\section{Experimental}

The heterosite sample was obtained from a private collection in the shape of a rock piece measuring a few $\mathrm{cm}^{2}$ across. It had been sampled from the Buranga pegmatite rock, Rwanda [8]. Crushed smaller pieces were finely ground in an agate mortar and the resulting powder was used for the subsequent measurements.

Powder X-ray diffraction (XRD) patterns were recorded with a Shimadzu XRD-6000 diffractometer using $\mathrm{FeK}_{\alpha}$ radiation and a graphite monochromator. The $2 \theta$ range between $5^{\circ}$ and $70^{\circ}$ was scanned at a speed of $0.5^{\circ} / \mathrm{min}$. Silicon was added to the sample powder as an internal standard. Unit-cell parameters were calculated by least-squares refinement after subtracting the background and the $\mathrm{K}_{\alpha 2}$ contribution and using intensity and angular weighting of the most intense peaks.

Microprobe analyses were carried out with a Jeol microscope, model JXA 8900R, equipped with a Noran EDS system and operating at $(20 \mathrm{kV}, 25 \mathrm{nA})$. A distinct well-developed crystallite, selected on the basis of the SEM observations, was probed at 20 different spots. Counting times were 10-100 s. Apatite, fayalite and rhodonite standards were used for the determinations of P (and Ca), Fe and Mn, respectively.

The Mössbauer spectra were collected in transmission geometry with WissEl time-mode spectrometers using triangular source motions and ${ }^{57} \mathrm{Co}(\mathrm{Rh})$ sources. All isomer shift values quoted hereafter are relative to $\alpha$-Fe at RT. The spectrometers, being of excellent linearity, were calibrated using Fe foil or standard $\alpha-\mathrm{Fe}_{2} \mathrm{O}_{3}$ powder depending on the applied velocity scale. The absorber-thickness was approximately $6 \mathrm{mg}$ Fe per $\mathrm{cm}^{2}$ and counts were accumulated in 1024 channels. The velocity increment per channel was $\sim 0.042 \mathrm{~mm} / \mathrm{s}$ for $T \leq 54 \mathrm{~K}$ (magnetic spectra) and $\sim 0.016 \mathrm{~mm} / \mathrm{s}$ for $T \geq 65 \mathrm{~K}$ (paramagnetic spectra). Final off-resonance counts were typically $\sim 10^{6}$ per channel. The temperature of the absorber was varied within the range 15-295 K using standard commercial cryogenic equipment. All spectra were collected with the absorber plane perpendicular with respect to the incident $\gamma$-ray beam because no asymmetry in the line intensities was observed from the spectra in the paramagnetic region, and hence texture effects were not occurring.

\section{Results}

\subsection{Characterization}

All peaks detected in the XRD pattern could be assigned to the orthorhombic structure of heterosite. Indexed in the Pmnb space group the unit-cell parameters, expressed in $\AA$, were found as $a=9.733(1), b=5.837$ (1) and $c=4.776$ (1). These parameters are in agreement with those reported in the literature for a sample with composition $\mathrm{Fe}_{0.65} \mathrm{Mn}_{0.35} \mathrm{PO}_{4}$, i.e., $a=9.79 \AA$, $b=5.83 \AA$ and $c=4.77 \AA$ [1]. The somewhat lower value for $a$ for the present heterosite phase

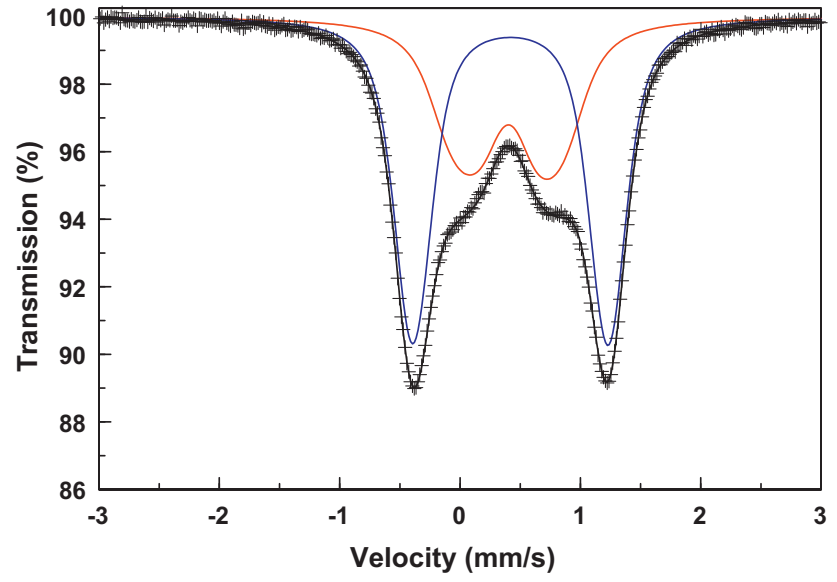

Fig. 1. Room temperature Mössbauer spectrum of the heterosite-containing rock sample from Buranga, Rwanda. Solid lines are the adjusted components and their superposition as calculated in terms of two model-independent quadrupolesplitting distributions. The sharp outer doublet is due to the heterosite phase.

might be related to the higher $\mathrm{Mn}^{3+}$ content (see hereafter) for this sample as compared to the sample considered by the authors of Ref. [1], $\mathrm{Mn}^{3+}$ (ionic radius $0.61 \AA$ ) being smaller than $\mathrm{Fe}^{3+}(0.64 \AA)$ and causing distortions of the M2 octahedra as a result of its Jahn-Teller activity.

The elemental contents measured at the different spots probed by the electron beam were found to be remarkably uniform and indicate a chemical composition of $\left(\mathrm{Fe}_{0.54} \mathrm{Mn}_{0.43} \mathrm{Mg}_{0.03}\right) \mathrm{PO}_{4}$, with subordinate traces of $\mathrm{Na}, \mathrm{K}, \mathrm{Ca}$ and $\mathrm{Al}$. It is expected that the small concentration of $\mathrm{Mg}$ in the sample does not significantly affect the results obtained from Mössbauer spectroscopy.

\subsection{Mössbauer spectra}

A typical Mössbauer spectrum as observed at all applied temperatures $T \geq 65 \mathrm{~K}$ is reproduced in Fig. 1 . Clearly, the spectrum consists of a broad inner doublet and a narrower outer doublet. These high-temperature, or paramagnetic, spectra were fitted in terms of a superposition of two unconstraint model-independent quadrupole-splitting distributions (QSD) [9]. The isomer shifts and the characteristics of the evaluated QSD profiles for the high- $\Delta E_{\mathrm{Q}}$ component at different temperatures are listed in Table 1 . Consistently, these profiles are almost perfectly symmetric as evidenced by the observation that their maximum-probability values, $\Delta E_{\mathrm{Qm}}$, are very close to their respective average values, $\Delta E_{\mathrm{Q}, a v}$, the mean

Table 1

Mössbauer parameters at various temperatures $T$ of the heterosite component as obtained from fitting the spectra with a superposition of two model-independent quadrupole-splitting distributions.

\begin{tabular}{rlllll}
\hline$T(\mathrm{~K})$ & $\delta^{\mathrm{a}}(\mathrm{mm} / \mathrm{s})$ & $\Delta E_{\mathrm{Q}, \mathrm{m}^{\mathrm{b}}}(\mathrm{mm} / \mathrm{s})$ & $\Delta E_{\mathrm{Q}, \mathrm{av}}{ }^{\mathrm{c}}(\mathrm{mm} / \mathrm{s})$ & $\sigma^{\mathrm{d}}(\mathrm{mm} / \mathrm{s})$ & $R A^{\mathrm{e}}$ \\
\hline 85 & 0.524 & 1.632 & 1.619 & 0.17 & 0.59 \\
105 & 0.517 & 1.638 & 1.629 & 0.18 & 0.58 \\
125 & 0.509 & 1.649 & 1.640 & 0.20 & 0.56 \\
155 & 0.496 & 1.631 & 1.630 & 0.17 & 0.59 \\
180 & 0.483 & 1.645 & 1.617 & 0.16 & 0.62 \\
204 & 0.470 & 1.617 & 1.636 & 0.18 & 0.58 \\
222 & 0.456 & 1.658 & 1.638 & 0.20 & 0.60 \\
252 & 0.441 & 1.648 & 1.642 & 0.19 & 0.58 \\
277 & 0.426 & 1.661 & 1.637 & 0.19 & 0.60 \\
295 & 0.413 & 1.626 & 1.625 & 0.16 & 0.63
\end{tabular}

a Isomer shift versus $\alpha$-Fe at room temperature (estimated error: $\pm 0.005 \mathrm{~mm} / \mathrm{s}$ ).

b Maximum-probability quadrupole splitting (estimated error: $\pm 0.015 \mathrm{~mm} / \mathrm{s}$ ).

c Average quadrupole splitting (estimated error: $\pm 0.02 \mathrm{~mm} / \mathrm{s}$ ).

d Second-order moment (estimated error: $\pm 0.015 \mathrm{~mm} / \mathrm{s}$ ).

e Relative spectral area (estimated error: \pm 0.02 ) 


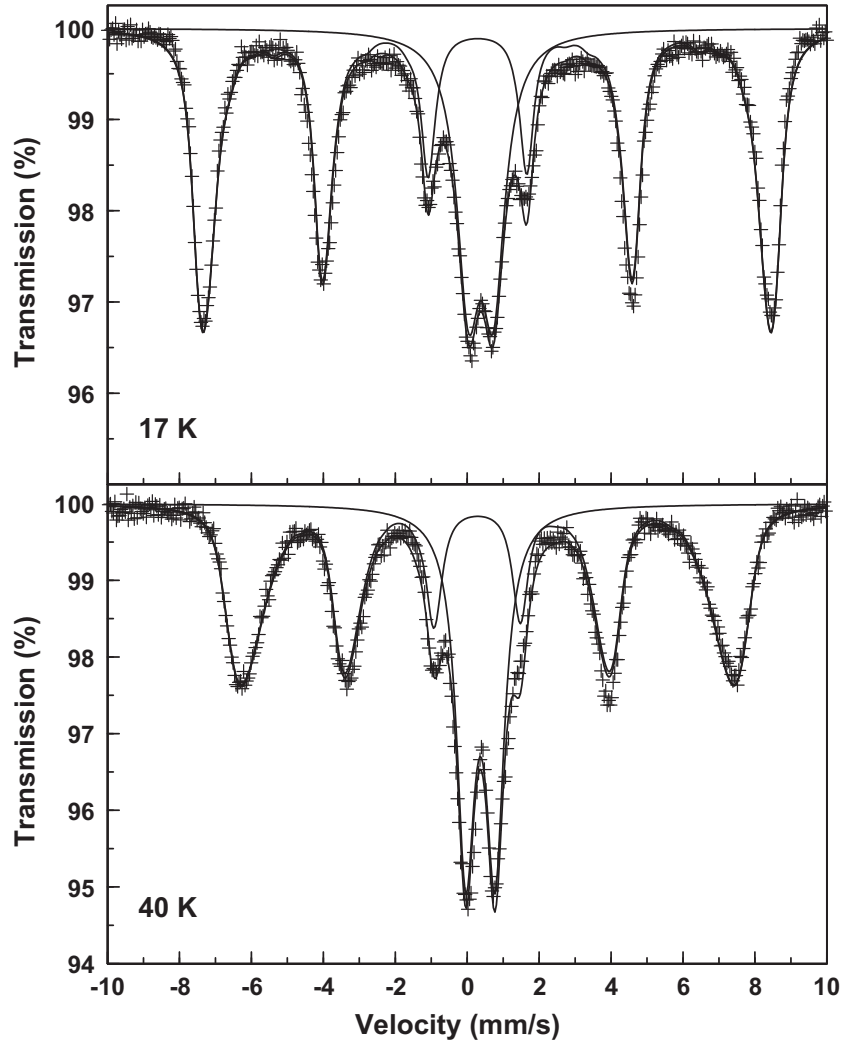

Fig. 2. Mössbauer spectra recorded at $17 \mathrm{~K}$ and $40 \mathrm{~K}$ of the heterosite-containing rock sample from Buranga, Rwanda. Solid lines are the adjusted components and their superposition. The sextet component is due to the heterosite phase and was fitted with a model-independent hyperfine field distribution.

difference being only $\sim 0.02 \mathrm{~mm} / \mathrm{s}$. Within experimental error limits, the quadrupole splitting is constant with temperature and its magnitude is unusually high for a ferric state.

In sharp contrast with the results for the high- $\Delta E_{\mathrm{Q}}$ components, the QSD profiles obtained for the low- $\Delta E_{\mathrm{Q}}$ components exhibit strong asymmetry with a long tail spreading towards the higher values in the distributions. The average values $\Delta E_{\mathrm{Q}, \mathrm{av}}$ are randomly scattered within the range $0.71 \pm 0.03 \mathrm{~mm} / \mathrm{s}$ and the relative contribution to the total spectral area varies within the range $(40 \pm 3) \%$. The isomer shift $\delta$ is $0.39 \mathrm{~mm} / \mathrm{s}$ at RT and $0.51 \mathrm{~mm} / \mathrm{s}$ at $80 \mathrm{~K}$. As discussed and argued in Section 4, the low- $\Delta E_{\mathrm{Q}}$ component is not due to $\mathrm{Fe}^{3+}$ cations in the heterosite structure, but to ferric cations that are present in some admixed $\mathrm{Fe}^{3+}$-bearing mineral species. As the low- and high- $\Delta E_{\mathrm{Q}}$ subspectra are well resolved, the presence of the admixed phase has no significant effect whatsoever on the Mössbauer parameters of the heterosite spectral contribution.

Two example Mössbauer spectra recorded at relatively low temperatures $(T<60 \mathrm{~K})$ are presented in Fig. 2 . Typically, the spectra in this low-temperature region are composed of a broad doublet superimposed on a broadened sextet, the width of the latter increasing with increasing temperature. Attempts to fit the spectra with a superposition of a model-independent ferric hyperfine-field distribution and a single quadrupole doublet failed in that the line shape of the sextet contributions could not be reproduced adequately, showing consistent and unacceptable misfits for all six absorption lines. The reason for this failure was found to be the large value of the quadrupole splitting of the magnetic component, reflecting the feature that the quadrupole interaction cannot be considered as a perturbation on the magnetic interaction. In such a case a magnetic subspectrum, either appearing as a single sextet or as an elemental sextet in a hyperfine-field distribution, cannot be
Table 2

Mössbauer parameters at various temperatures $T$ of the heterosite phase as obtained from fitting the spectra with a superposition of a central quadrupole doublet and model-independent hyperfine-field distribution.

\begin{tabular}{llllllll}
\hline$T(\mathrm{~K})$ & $\delta^{\mathrm{a}}(\mathrm{mm} / \mathrm{s})$ & $B_{\mathrm{hf,m}}^{\mathrm{b}}(\mathrm{kOe})$ & $1 / 2 e^{2} q Q^{\mathrm{c}}(\mathrm{mm} / \mathrm{s})$ & $\eta^{\mathrm{d}}$ & $\Omega^{\mathrm{e}}\left(^{\circ}\right)$ & $\Psi^{\mathrm{f}}\left({ }^{\circ}\right)$ & $\mathrm{RA}^{\mathrm{g}}$ \\
\hline 17 & 0.543 & 473 & 1.50 & 0.79 & 40 & 93 & 0.67 \\
20 & 0.540 & 463 & 1.46 & 0.78 & 40 & 89 & 0.66 \\
25 & 0.536 & 457 & 1.48 & 0.83 & 40 & 91 & 0.66 \\
29 & 0.534 & 445 & 1.49 & 0.70 & 41 & 90 & 0.65 \\
35 & 0.537 & 441 & 1.50 & 0.79 & 40 & 94 & 0.64 \\
40 & 0.529 & 401 & 1.52 & 0.93 & 40 & 68 & 0.63 \\
46 & 0.534 & 375 & 1.58 & 0.95 & 41 & 60 & 0.62 \\
49 & 0.536 & 359 & 1.57 & 0.88 & 42 & 59 & 0.60 \\
54 & 0.546 & 303 & 1.54 & 0.84 & 43 & 55 & 0.58 \\
57 & 0.589 & 250 & 1.50 & 0.79 & 43 & 60 & 0.55
\end{tabular}

a Isomer shift versus $\alpha$-Fe at room temperature (estimated error: $\pm 0.01 \mathrm{~mm} / \mathrm{s}$ ).

b Maximum-probability hyperfine field (estimated error: $\pm 3 \mathrm{kOe}$ ).

c (Average) quadrupole coupling constant (estimated error: $\pm 0.03 \mathrm{~mm} / \mathrm{s}$ ).

d Asymmetry parameter of the electric field gradient EFG (estimated error: \pm 0.05 ).

e Zenithal angle of the hyperfine field in the EFG $(x, y, z)$-axes frame (estimated error: $\pm 5^{\circ}$ ).

${ }^{\mathrm{f}}$ Azimuthal angle of the hyperfine field in the EFG $(x, y, z)$-axes frame (estimated error: $\pm 15^{\circ}$ )

g Relative spectral area (estimated error: \pm 0.02 ).

approximated by a summation of six Lorentzian lines of which the positions $v_{0, i}$ are linear functions of the hyperfine parameters $\delta$ (isomer shift), $\varepsilon_{\mathrm{Q}}$ (quadrupole shift, related to the quadrupole splitting $\left.\Delta E_{\mathrm{Q}}\right)$ and $B_{\mathrm{hf}}$ (the magnetic hyperfine field): $v_{0, i}=\delta+\alpha_{i} \varepsilon_{\mathrm{Q}}+\beta_{i} B_{\mathrm{hf}}$, $i=1, \ldots, 6$ [9]. Instead, the numerical analyses has to be based on the complete hyperfine-interaction Hamiltonian, the diagonalization of which allows the calculation of the energies and probabilities of the eight transitions between the sublevels of the nuclear ground state and those of the first excited state of ${ }^{57} \mathrm{Fe}[10,11]$. This procedure is seldom to be used in the case of $\mathrm{Fe}^{3+}$ magnetic spectra, but is commonly required in the case of $\mathrm{Fe}^{2+}$.

The solid lines in Fig. 2 represent the two components (doublet + sextet) and their superposition as fitted to the experimental data. For the doublet contribution a single quadrupole doublet was considered, while for the magnetic component a model-independent hyperfine-field distribution was modelled. The quadrupole splitting of the doublet was fitted at a value scattered in the range $0.75 \pm 0.05 \mathrm{~mm} / \mathrm{s}$ for all 10 -recorded low-temperature spectra. For the magnetic component the following parameters had to be adjusted: isomer shift $\delta$, quadrupole coupling constant $1 / 2 e^{2} q Q$ asymmetry parameter $\eta$ of the electric field gradient (EFG), the polar angles $\Omega$ and $\psi$ of the direction of the hyperfine field in the principal axes frame of the EFG (see Fig. 4 in Ref. [2]) and the relative spectral area $R A$. The obtained values for these parameters are listed in Table 2 for the various temperatures applied in this study. It may be reminded at this point that the relation between the quadrupole coupling constant and the quadrupole splitting $\Delta E_{\mathrm{Q}}$ as reflected in a paramagnetic doublet spectrum is given by:

$\Delta E_{\mathrm{Q}}=\frac{1}{2}(e q)(e Q) \sqrt{1+\frac{\eta^{2}}{3}}$,

with $e Q$ the nuclear quadrupole moment and eq (or $V_{z z}$ ) the principal component of the EFG. Further, the asymmetry parameter $\eta$ is defined by $\eta=\left|V_{x x}-V_{y y}\right| / V_{z z}$, with $V_{i i}$ the diagonal elements of the EFG tensor so organized that $V_{z z}>V_{x x} \geq V_{y y}$.

\section{Discussion}

It is obvious that the broad inner doublet observed in the paramagnetic Mössbauer spectra and the doublet appearing in the low-temperature spectra arise from a same type of Fe site. As it is generally accepted that in heterosite-purpurite solid solutions the $\mathrm{Fe}^{3+}$ and $\mathrm{Mn}^{3+}$ cations exclusively occupy the M2 octahedra, 
that type of iron site is not part of the heterosite structure and thus this finding suggests that the sample used for this study is not a pure heterosite species. One could argue that there may occur heterosite species for which for some reason part of the cations have entered the M1 sites in the structure. However, if this were true the $\mathrm{M} 1$ cations would take part in the magnetic ordering and give rise to a second sextet component in the low-temperature Mössbauer spectra. It is therefore concluded that the low $-\Delta E_{\mathrm{Q}}$ doublet is due to an iron-bearing impurity phase. Since the XRD pattern does not show the presence of a significant amount of a second phase beside heterosite, it is suggested that the impurity phase is amorphous. This suggestion is consistent with the values of the observed very broad distribution of the quadrupole splitting. The presence of the impurity spectral component does not interfere with the heterosite subspectrum in the sense that the characteristic Mössbauer parameters of the latter are well resolved and their determination not obscured. This means that the present Mössbauer results are useful and valuable for deriving some basic properties of the mineral heterosite. It should be noted at this point that the occurrence of amorphous manganese-iron phosphate has been reported for other pegmatite rocks [12]. Also, Fransolet [13] has frequently noticed, under the petrographic microscope, that an isotropic mineral occurs in heterosite specimens, including those of the Buranga mine. He concludes that this mineral is an amorphous phosphate rich in Fe.

From Tables 1 and 2, and considering Eq. (1), it is noticed that the values of the quantity $1 / 2 e^{2} q Q$ obtained from the low-temperature spectra and those of the quadrupole splitting, $\Delta E_{\mathrm{Q}, \mathrm{m}}$ or $\Delta E_{\mathrm{Q}, \mathrm{av}}$, derived from the high-temperature spectra are reasonably well in line. The results further indicate that the quadrupole interaction is nearly invariant with temperature, which is a common feature for $\mathrm{Fe}^{3+}$ compounds. Yamada and Chung [4] examined the RT Mössbauer spectra for synthetic solid solutions $\mathrm{Mn}_{y} \mathrm{Fe}_{1-y} \mathrm{PO}_{4}$. They found a clear linear correlation between the quadrupole splitting $\Delta E_{\mathrm{Q}}$ and the Mn content $y$. According to this correlation, the presently observed $\Delta E_{\mathrm{Q}}$ value of $1.625 \mathrm{~mm} / \mathrm{s}$ for the heterosite phase (Table $1, T=295 \mathrm{~K}$ ) corresponds to an Mn content of $y \approx 0.40$, which is in reasonable agreement with the earlier derived composition (Section 3).

The existence of a distribution of hyperfine field values for the heterosite phase is explained by the non-ordered distribution of the $\mathrm{Fe}^{3+}$ and $\mathrm{Mn}^{3+}$ cations among the $\mathrm{M} 2$ sites. This feature implies that the local cation environment of the probe iron nuclei is not uniform and that consequently the molecular field, and hence the strength of the hyperfine field, acting at the ${ }^{57} \mathrm{Fe}$ probes differ from one site to the other. It is quite possible that also the direction of the magnetic hyperfine field is not unique. The values obtained for the polar angles are therefore to be considered as average values. It might be that the assumption of one single value for the angle $\Omega$ explains the minor, but consistent misfits of the calculated line shapes, in particular in the velocity range of the fifth absorption lines (see Fig. 2). The second polar angle $(\Psi)$ in general has only a weak influence on the obtained theoretical line shape. It is therefore not conclusive whether the sudden drop from $\Psi \approx 90^{\circ}$ to $\Psi \approx 60^{\circ}$ observed at temperatures around $40 \mathrm{~K}$ is real. On the other hand, the angle $\Omega$ between the hyperfine field and the EFGs principal axis is usually rather well defined. The obtained results show that in the case of heterosite $\Omega$ does not change with temperature and remains at $\sim 40^{\circ}$. The orientation of the hyperfine field with respect to the crystallographic axes of the orthorhombic unit cell, however, cannot be inferred from the present data concerning the polar angles.

The temperature variation of the maximum-probability hyperfine field, $B_{\mathrm{hf}}(T)$, is presented in Fig. 3 and has been interpreted by the model developed by Bean and Rodbell [14]. This model is based on the classical molecular field concept [15], but additionally takes into account that the strength of the magnetic exchange

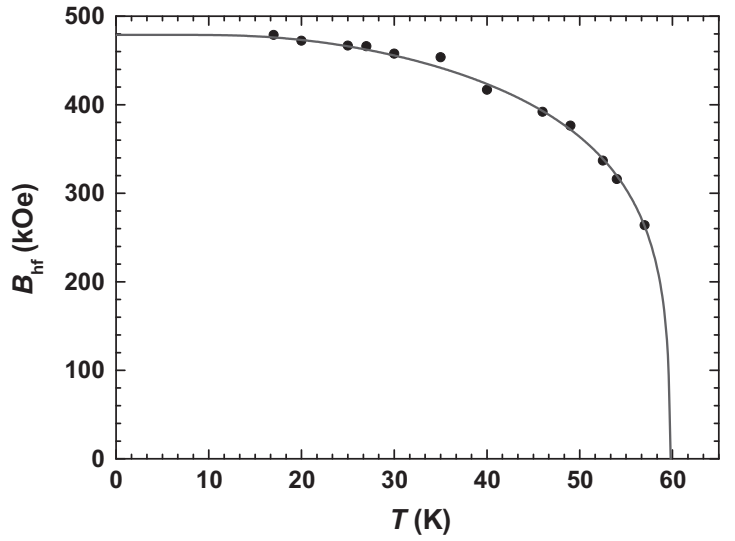

Fig. 3. Temperature variation of the hyperfine field $B_{\mathrm{hf}}$ of heterosite. Solid line was calculated in terms of the Bean-Rodbell model. The error bars are approximately half the size of the curve symbols.

interactions varies within the magnetically ordered regime as a result of magnetostriction. This effect is thought to be especially important when deformable lattices are concerned. Due to this magnetostriction effect, the inter-atomic distances change with changing sublattice magnetization, and consequently the strength of the magnetic interactions also change.

In terms of the Bean-Rodbell model, $B_{\mathrm{hf}}(T)$ follows a Brillouin behaviour described by:

$B_{\mathrm{hf}}(T)=B_{\mathrm{hf}}(0) \times B_{S}(x)$

with $B_{\mathrm{hf}}(0)$ the saturation value, $B_{S}(x)$ the Brillouin function for magnetic spin moment $S\left(S=5 / 2\right.$ for $\left.\mathrm{Fe}^{3+}\right)$ and with argument $x$ given by:

$x=\left[2 a+4 b \frac{B_{\mathrm{hf}}^{2}(T)}{B_{\mathrm{hf}}^{2}(0)} \eta_{B R}\right] \frac{T_{\mathrm{N}}}{T} \frac{B_{\mathrm{hf}}(T)}{B_{\mathrm{hf}}(0)}$,

with

$a=\frac{3 S}{2(S+1)}$

and

$b=\frac{9\left[(2 S+1)^{4}-1\right]}{20[2(S+1)]^{4}}$

In Eq. (3), the quantity $\eta_{\mathrm{BR}}$ is a measure for the departure from an ideal second-order magnetic transition. Its value is generally structure-related and basically determined by the compressibility of the lattice and, as a consequence, of the deformation of the lattice on reaching the magnetic transition [14]. In case of an ideal second-order transition the value for $\eta_{\mathrm{BR}}$ equals zero (rigid lattice) and the variation of $B_{\mathrm{hf}}(T)$ then follows the classical Brillouin curve. Values for $\eta_{\mathrm{BR}}$ exceeding 1 indicate first-order transitions, and values in between 0 and 1 are to be considered as intermediateorder transitions for which the transition phenomena are not well understood.

The solid curve in Fig. 1 shows the calculated temperature dependence of the hyperfine field. It is the result of recursive calculations embedded in a least-squares iteration routine in which $\eta_{\mathrm{BR}}, B_{\mathrm{hf}}(0)$ and $T_{\mathrm{N}}$ were adjustable parameters. Their values were iterated as $0.90,479 \mathrm{kOe}$ and $59.7 \mathrm{~K}$, respectively. The agreement between the calculated and observed temperature variations is obviously satisfactory. The value for $\eta_{\mathrm{BR}}$ of 0.90 infers that the magnetic transition in heterosite is close to a first-order transition. It should be mentioned at this point that similar features regarding the $B_{\mathrm{hf}}(T)$ variations have recently been noticed by some of the present authors for the ferric hyperfine fields acting at the two 


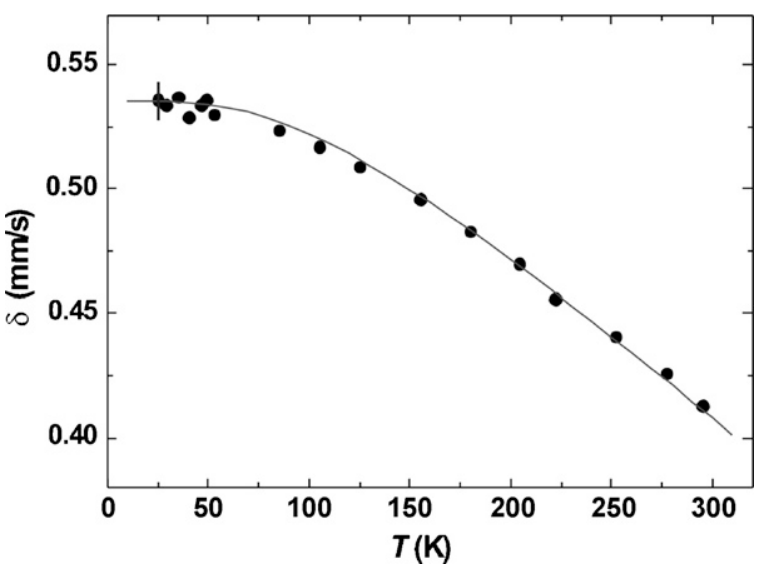

Fig. 4. Temperature variation of the isomer shift $\delta$ of heterosite. Solid line is the theoretical variation as obtained by applying the Debye model for the lattice vibrational spectrum to calculate the second-order Doppler shift as a function of temperature. The shown error bar refers to the low-temperature data $(T<60 \mathrm{~K})$. For the hightemperature data the error bars are approximately half of that.

octahedral sites in a synthetic leucophosphite sample [16] and for the ferrous hyperfine field in a synthetic szomolnokite [17] and in a synthetic triphylite [2], yielding values for $\eta_{\mathrm{BR}}$ of 0.63 , 0.74 and 0.97 , respectively. It is not clear whether the difference in $\eta_{\mathrm{BR}}$ values for all these materials can be related to structural or cationic charge differences. More quantitative data about the minerals, such as compressibility, fractional volume change at the magnetic transition temperature, slope of the variation of the transition temperature with the lattice volume, etc. should be known in order to allow an at least qualitative interpretation of obtained $\eta_{\mathrm{BR}}$ parameters.

The temperature dependence of the isomer shift $\delta$ is plotted in Fig. 4. The data have been interpreted on the basis of the expression: $\delta(T)=\delta_{\mathrm{I}}+\delta_{\text {SOD }}$. The intrinsic isomer shift $\delta_{\mathrm{I}}$ is determined by the selectron density at the nucleus and in a first approximation exhibits a linear temperature variation: $\delta_{\mathrm{I}}(T)=\delta_{\mathrm{I}}(0)+\alpha \times 10^{-5} T$. This weak variation, however, has only significant effect on $\delta(T)$ when data at temperatures far exceeding RT are involved. In contrast, the second-order Doppler shift, $\delta_{\text {SOD }}$, is strongly dependent on temperature, being related to the vibrational properties of the probe nuclei in the crystal structure. The Debye approximation for the lattice vibrational spectrum provides an adequate model for calculating $\delta_{\text {SOD }}[18,19]$. It contains one parameter, the so-called characteristic Mössbauer temperature, $\Theta_{\mathrm{M}}$, the value of which is obtained by adjusting the theoretical expression for $\delta(T)$ to the experimental values. The solid curve in Fig. 4 represents the calculated temperature variation with $\delta_{\mathrm{I}}=(0.64 \pm 0.01) \mathrm{mm} / \mathrm{s}$ and $\Theta_{\mathrm{M}}=(400 \pm 20) \mathrm{K}$. This latter value does not fit the general tendency that ferric Mössbauer temperatures are close to or exceeding $500 \mathrm{~K}$, while ferrous species commonly exhibit values close to $400 \mathrm{~K}[18,19]$. It is tempting to ascribe the low value of $\Theta_{M}$ for heterosite to the defect nature of its structure. That suggestion, however, remains speculative. For the aforementioned reason, the thermal coefficient $\alpha$ could not be determined. The value of $\delta_{\mathrm{I}}$ was found to be $0.64 \mathrm{~mm} / \mathrm{s}$.

\section{References}

[1] W. Eventoff, R. Martin, D.R. Peacor, American Mineralogist 57 (1972) 45-51.

[2] A. Van Alboom, E. De Grave, M. Wohlfahrt-Mehrens, American Mineralogist 96 (2011) 408-416.

[3] A.S. Andersson, B. Kalska, L. Häggström, J.O. Thomas, Solid State Ionics 130 (2000) 41-52.

[4] A. Yamada, S.C. Chung, Journal of the Electrochemical Society 148 (2001) A960-A967.

[5] H.J. Tan, J.L. Dodd, B. Fultz, Journal of Physical Chemistry C 113 (2009) $2526-2531$.

[6] Th. Fehr, R. Hochleitner, A. Laumann, E. Schmidbauer, J. Schneider, Physics and Chemistry of Minerals 37 (2010) 179-189.

[7] L. Aldon, A. Perea, M. Womes, C.M. Ionica-Bousquet, J.-C. Jumas, Journal of Solid State Chemistry 183 (2010) 218-222.

[8] V.D.C. Daltry, O. von Knorring, Geologica Belgica 1 (1998) 9-15.

[9] R.E. Vandenberghe, E. De Grave, P.M.A. de Bakker, Hyperfine Interactions 83 (1994) 29-49.

[10] W. Kündig, Nuclear Instruments and Methods in Physics Research 48 (1967) 219-228.

[11] G.R. Hoy, S. Chandra, Journal of Chemical Physics 47 (1967) 961-965.

[12] M.J. Gallagher, Mineralogical Magazine 36 (1967) 50-59.

[13] A.-M. Fransolet, private communication.

[14] C.P. Bean, D.S. Rodbell, Physical Review 126 (1962) 104-115.

[15] A.H. Morrish, The Physical Principles of Magnetism, Wiley, New York, 1965.

[16] V.G. de Resende, G.M. da Costa, E. De Grave, A. Van Alboom, American Mineralogist 93 (2008) 483-487.

[17] A. Van Alboom, E. De Grave, V.G. de Resende, J.A.M. Gómez, Journal of Molecular Structure 924 (2009) 448-456.

[18] E. De Grave, A. Van Alboom, Physics and Chemistry of Minerals 18 (1991) 337-342.

[19] S.G. Eeckhout, E. De Grave, Physics and Chemistry of Minerals 30 (2003) $142-146$. 